56 ENDOCARDIAL LEFT VENTRICULAR PACING ACROSS THE INTER-VENTRICULAR SEPTUM FOR CARDIAC RESYNCHRONISATION THERAPY -- CLINICAL RESULTS

${ }^{1}$ James Gamble*, ${ }^{1}$ Neil Herring, 'Matthew Ginks, ${ }^{1}$ Kim Rajappan, ${ }^{1}$ Yaver Bashir, ${ }^{2}$ Timothy Betts. ${ }^{1}$ Oxford University Hospitals NHS Foundation Trust, ${ }^{2}$ Oxford University Hospitals NHS Trust; *Presenting Author

\subsection{6/heartjnl-2016-309890.56}

Introduction Cardiac Resynchronisation Therapy (CRT) is an effective therapy for selected patients with heart failure, but is limited in some patients by inability to place the left ventricular (LV) lead via the coronary sinus. We have developed an alternative technique, placing the LV lead endocardially through an interventricular septal puncture. We now report the results of a pilot study assessing this technique, and in addition assessing the optimal endocardial pacing site.

Methods All patients were anticoagulated with INR 2.5-3.5. A superior approach ventricular transseptal puncture using RF energy was performed. We undertook endocardial LV electrical activation mapping. Acute haemodynamic response (AHR) to CRT was assessed at the site of latest electrical activation (LEA), at the site of latest mechanical activation (LMA) as assessed on pre-procedure speckle-tracking echocardiography, and at an empirical lateral position. An active-fixation pacing lead was delivered through the septum to the site with optimal haemodynamics.

Patients were followed up at 6 months with the Packer Clinical Composite Score (CCS) which categorises HF patients as clinically improved or worsened. AHR results were compared with generalised mixed-effects multi-level linear modelling.

Results 18 patients were recruited, 13 with failed transvenous LV lead placement and 5 non-responders to standard CRT with poor LV lead position. Age was $66 \pm 12,83 \%$ male, QRSD $156 \pm 14 \mathrm{~ms}$, ischaemic 44\%, NYHA class $2.9 \pm 0.6$, EF $28 \pm 7 \%$, CHADS-VASc score $3.6 \pm 1.4$ (all mean+/-SD).

AHR could be assessed in 17 patients; the optimal site was at the LEA in 8, at the LMA in 6 and lateral in 2. Overall there was no significant difference in AHR between the LEA and LMA. Electrical delay $>100 \mathrm{~ms}$ was a significant multivariate predictor of AHR, but placement at the LMA site was not.

The procedure was successful in all and safe with no serious complications, and two wound haematomas.

Packer clinical composite score improved at 6 months in $57 \%$, and was worse in $43 \%$. LV ejection fraction improved $>5 \%$ in $71 \%$, from $28+/-7 \%$ to $39+/-9 \%$. 6 min walking distance improved $>10 \%$ in $75 \%$, from $256+/-128 \mathrm{~m}$ to $314+/-$ $111 \mathrm{~m}$.

One patient suffered a lacunar ischaemic stroke after 5 months with partial neurological recovery, associated with labile INRs. Another died of pneumonia after 3 months.

Conclusions LV endocardial pacing via interventricular septal puncture in patients in whom standard CRT is not possible is similarly effective and durable, with reasonable risks. Optimal pacing sites may need personalisation to the patient, but a lateral position with a long electrical delay is a reasonable empirical site.

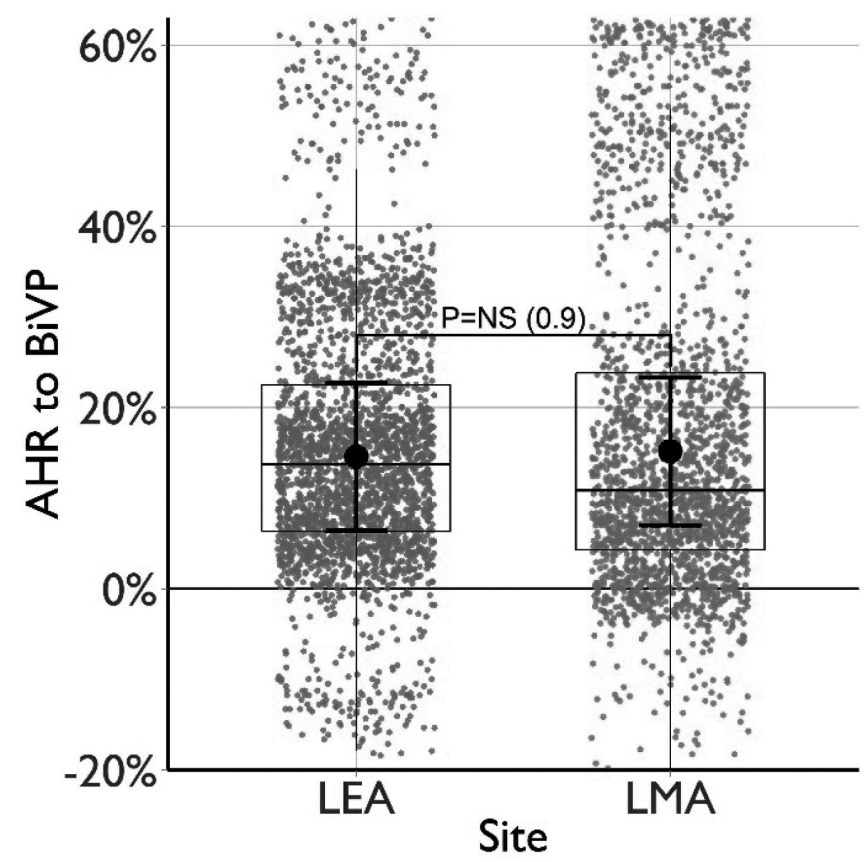

Abstract 56 Figure 1 Acute haemodynamic response to biventricular pacing at the sites of latest electrical and mechanical activation

\section{IMPROVING THE USE OF REMOTE ELECTROCARDIOGRAPHIC MONITORING IN HOSPITAL INPATIENTS: A QUALITY IMPROVEMENT PROJECT}

${ }^{1}$ Richy Morais, ${ }^{1}$ Amrita Randhawa*, ${ }^{2}$ Richard Dobson, ${ }^{1}$ Victor Chong. ${ }^{1}$ NHS Ayrshire and Arran; ${ }^{2}$ University of Glasgow; *Presenting Author

\subsection{6/heartjnl-2016-309890.57}

Introduction Remote electrocardiographic monitoring ("telemetry") is a useful tool to identify life threatening arrhythmic complications in hospital inpatients. However, whilst appropriate use criteria (AUC) exist, the application of them is not universal, thus leading to potentially ineffective and inefficient use of this limited resource. We aimed to establish the current

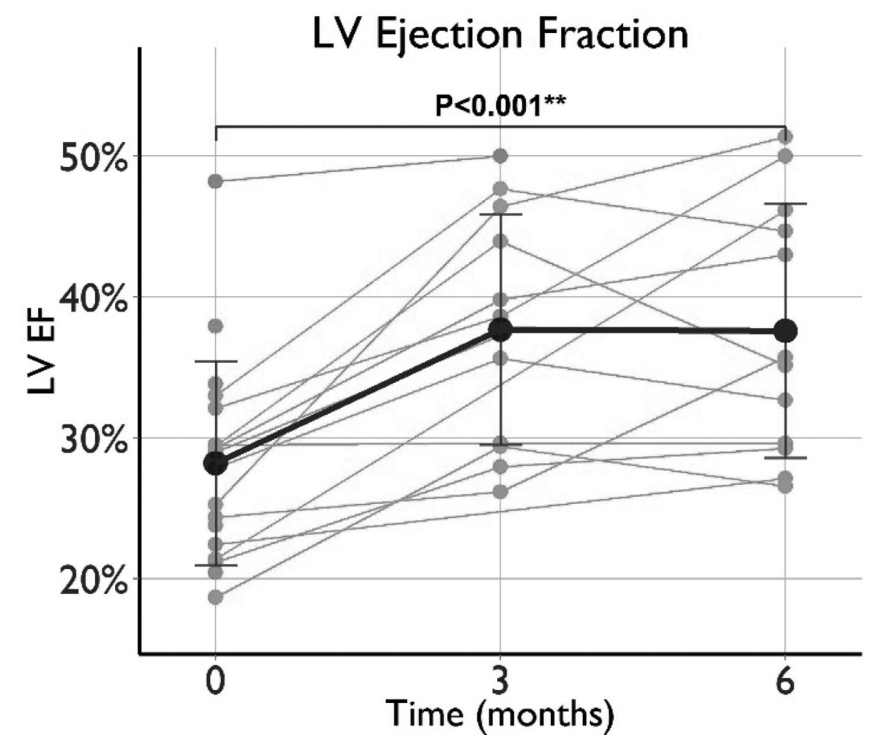

Abstract 56 Figure 2 Response to CRT as assessed by LVEF 
level of appropriate use of telemetry in hospital inpatients in our centre, and hypothesised that a new request card in addition to an educational program for requesting clinicians would help to improve adherence to published AUC.

Methods Prospective single centre quality improvement project in a 625-bed district general hospital, with approval from the local clinical governance committee. Initial data collection was performed over an 8 week period from March to May 2013, and included all patients for whom telemetry was requested for. Data collected included basic demographic and clinical information, the indication for telemetry, and the duration and results of the period of monitoring. The proposed indication was compared with the rating system documented in the American Heart Association 2004 consensus statement for remote electrocardiographic monitoring, ${ }^{1}$ and classed as level 1 (definitely indicated), level 2 (possibly indicated), or level 3 (not indicated). Following the initial data collection, the telemetry request form was redesigned to better reflect the AUC, and a series of seminars were held for junior clinicians on the use of telemetry at the same centre. The data collection was then repeated over another 8 week cycle from April to June 2015, and the results compared.

Results There were 48 patients (29 female) in cycle 1 and 51 patients $(27$ female) in cycle 2 . Mean age was $73.6 \pm 12.7$ years in cycle 1 and $69.9 \pm 17.8$ years in cycle $2(p=$ 0.231 ), and mean duration of recording was $3.58 \pm 2.90$ days in cycle 1 and $2.84 \pm 1.93$ days in cycle $2(p=0.439)$. The commonest indication in both data cycles was the identification of an electrolyte abnormality. Only one patient (in cycle 1) experienced a life threatening arrhythmia whilst on telemetry. In cycle 1 we identified that only $50 \%$ of requests were class 1 , and this improved to $75 \%$ in cycle 2 ( $p=$ 0.042 ; Figure 1). Additionally there were fewer gaps in recording in cycle 2 (mean $7.1 \pm 7.9 \mathrm{~h}$ versus $11.6 \pm 10.2$ $\mathrm{h}, p=0.030$ ).

Conclusions Whilst this is a single centre study with small numbers, we have shown that adherence to AUC for the use of telemetry in our hospital increased significantly following two simple, easy-to-implement, low cost initiatives. Sustaining such an improvement, and applying similar methods to

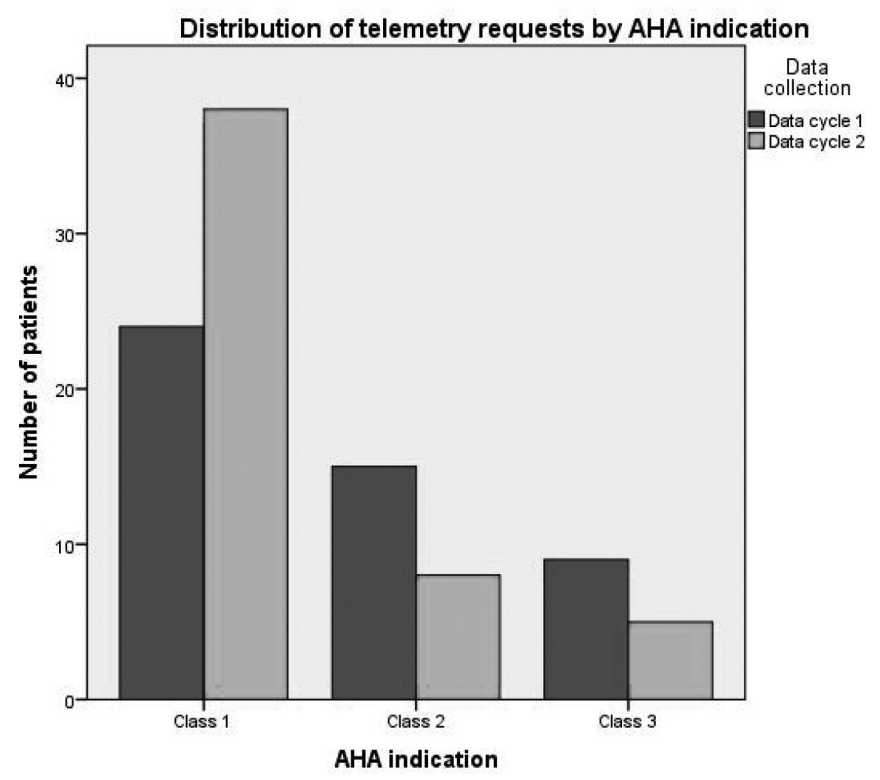

Abstract 57 Figure 1 Distribution of telemetry requests by AHA indication improve the appropriate use of other investigations, merits further study.

\section{REFERENCE}

1 Drew B, Califf R, Funk $M$, et al. Practice standards for electrocardiographic monitoring in hospital settings. Circ. 2004;110:2721--46

\section{ECG ELECTRODE POSITIONING -- LUCK OR JUDGMENT?}

${ }^{1}$ Oomesh Kishto*, 'Michael Pope, ${ }^{1}$ Alexander Hobson, ${ }^{2}$ Graham Petley, ${ }^{1}$ Michelle Evans, ${ }^{2}$ Richard Bain. ${ }^{1}$ Portsmouth NHS Trust; ${ }^{2}$ University of Southampton; * Presenting Author

\subsection{6/heartjnl-2016-309890.58}

Introduction Electrocardiography is the most common tool used to assist in the evaluation of cardiac disease. A standardised technique is crucial to allow accurate interpretation and comparison of serial tracings. Guidelines are produced by The American Heart Association and the Society for Cardiological Science and Technology. We sought to evaluate knowledge of precordial lead positioning amongst healthcare staff involved in the emergency care of patients with suspected cardiac disease.

Methods 106 staff of various groups were asked to identify the correct locations for precordial lead position on a schematic of the chest wall. Results were analysed according to distance from the correct position in millimeters $(\mathrm{mm})$ and a mean distance across all leads was calculated. Cardiac physiologists receive the most robust training in ECG acquisition and secondary analysis compared positions identified by other healthcare staff to physiologists. The $95 \%$ confidence interval for the physiologists' lead positions was calculated and then used to define the correct position for a comparison against other professional groups.

Results Of the 106 staff members recruited, 9\% were cardiac physiologists, $12 \%$ cardiographers, $11 \%$ cardiology nurses, $11 \%$ cardiology consultants or registrars, $18 \%$ non-cardiology nurses, $11 \%$ healthcare support workers, 13\% non-cardiology physicians, and $12 \%$ paramedics.

Knowledge of lead position was highly variable with many staff suggesting positions a significant distance from the guideline recommendations. Figure 1 represents the distribution of positions identified for each precordial lead. All of the leads tend be placed higher up the chest wall with V1, V2 and V6 also tending to be more lateral.

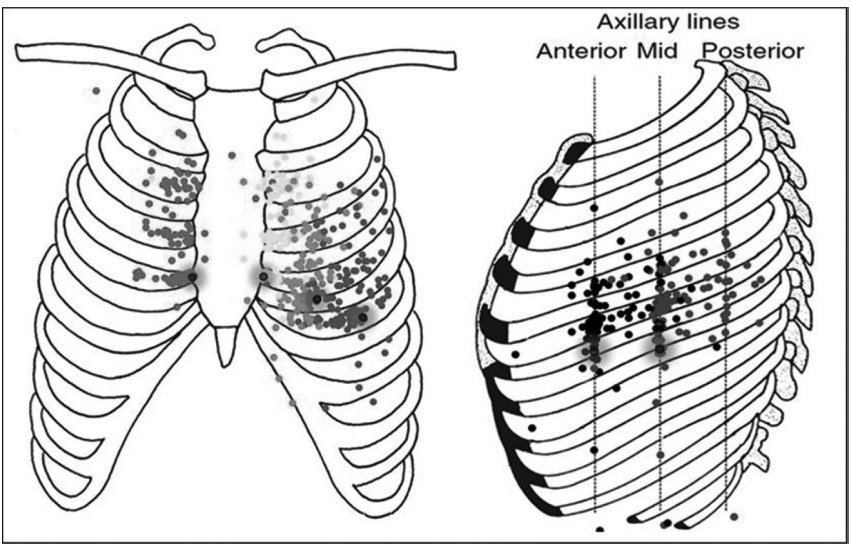

Abstract 58 Figure 1 Schematic illustration of electrode positions identified by the study cohort 